\title{
Deterministic method for obtaining nominal and uncertainty models of CD drives
}

\author{
E. Vidal, J. Stoustrup, P. Andersen, T.S. Pedersen, \\ Dept. of Control Engineering \\ Aalborg University \\ DK 9220 Aalborg \\ email: $\{$ enrique, jakob,pa, tom\}econtrol.aue.dk,
}

\author{
H.F. Mikkelsen \\ Bang \& Olufsen a/s \\ Peter Bangs vej 15 \\ DK 7600 Struer \\ hemobang-olufsen.dk
}

\begin{abstract}
In this paper a deterministic method for obtaining the nominal and uncertainty models of the focus loop in a CD-player is presented based on parameter identification and measurements in the focus loop of 12 actual $C D$ drives that differ by having worst-case behaviors with respect to various properties. The method provides a systematic way to derive a nominal average model as well as a structured multiplicative input uncertainty model, and it is demonstrated how to apply $\mu$-theory to design a controller based on the models obtained that meets certain robust performance criteria.
\end{abstract}

\section{Introduction}

Optical Disc Drives (ODD) are mainly characterized by the absence of the physical contact between the pick-up and the disc. Feedback control is necessary to control the position of the focus point of the laser in order to read the data. Two main control loops can be identified: the focus loop which maintains the focus point of the laser on the signal layer, and the radial loop which follows the track. Since the compact disc player was introduced in the market two decades ago, more products based in the same technology have been developed, as CD-ROM and DVD players having a track pitch of $1.6 \mu \mathrm{m}$ and $0.78 \mu \mathrm{m}$ respectively. Recently a new standard has been announced in Tokyo (february 2002), the socalled blue ray disc with a track pitch of $0.32 \mu \mathrm{m}$. The trend is clear, towards higher storage capacity and data transfer rate. The performance requirements to the position controllers have therefore increased at the same time. In parallel to the development of ODD's, much effort has been spent to solve the multivariable robustness analysis and synthesis where different classes of uncertainties have been considered. Unstructured uncertainties (full-block complex perturbation uncertainties) can be used in the $\mathcal{H}_{\infty}$ framework, see [DGKF89]. In general, a less conservative controller is achieved if the control problem is formulated in the $\mu$ framework which considers structured uncertainties. Common to both approaches is the description of model uncertainties as transfer functions, which are norm-bounded but otherwise unknown. Extensive literature can be found explaining how to formulate multivariable robustness problems but usually the nominal and the uncertainty models are assumed to be known. In a more realistic situation, the designer may only have a set of complex points in the Nyquist plane from several worst-case plants. Having a limited knowledge of the plant, it might not be trivial how to obtain a nominal and uncertainty models suited for the robust control framework. A possibility is to circumscribe the set of complex points of the different plants with discs at each frequency point. The center of the discs can be fitted to a rational transfer function yielding the nominal model and the radii of the circles represent the uncertainty model. This procedure is conservative as the obtained uncertainty model will introduce possible plants that are not present in the original set. Inherently, conservatiness in an uncertain model results in reduced performance. However, in view of the fact that the performance requirements of the ODD's positioning controllers are constantly increasing, this is unfortunate. In this paper a deterministic method for obtaining the nominal and uncertainty models of the focus loop in a CD-player is proposed, which is less conservative than the approach mentioned above. A thorough treatment of robust control approaches to $\mathrm{CD}$ control can be found in [SSB96]. Actually, two entire theses has been dedicated to this subject, see [Lee98, Det01]. The remainder of this paper is organized as follows. In Section 2 it is described how empirical models are derived from experiments on a number of different $\mathrm{CD}$ drives. These empirical models are exploited in Section 3 to derive both nominal and uncertainty models. The nominal model and the structured uncertainty model are in turn used in Section 4 for compensator synthesis. The synthesized compensators are evaluated in simulation in Section 5 . Finally, the findings of this paper are summarized in Section 6.

\section{Set of Nyquist points in 12 CD players}

The optical pick-up is a 2-axis device, enabling a movement of the lens in two axes: vertically for focus correction and horizontally for track following. Two coils which are orthogonal to each other are suspended between permanent magnets. A current through a coil creates a magnetic field which interacts with the magnetic field from the permanent 
magnet and the coil and consequently the lens will move in the corresponding direction. In this paper it has been chosen to study the focus actuator only. Similar results, however, transfer immediately to the radial loop, which has essentially the same dynamics. Fig. 1 shows the focus loop bode plot of 12 specific $C D$ drives which have been chosen from a larger set by extreme property behavior.
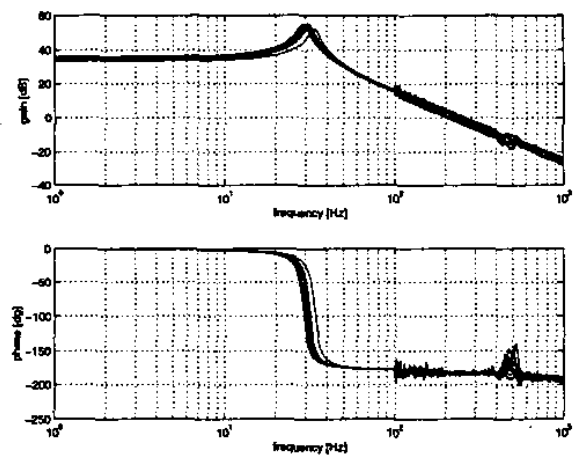

Figure 1: Empirical models of $12 \mathrm{CD}$ drives.

Two different methods have been employed in combination in order to obtain the bode plots, see [VSA ${ }^{+} 01$ ], yielding 12 focus open loop bode plots. Thus, the models used in this paper are linear. A method for modeling some of the nonlinearities is described in [YP00]. Another approach for linear modeling of CD mechanisms is given in [PGRV92].

From fig. 1 it can be seen that the resonance peak around $30[\mathrm{~Hz}]$ varies in frequency. The parasitic dynamics around $500[\mathrm{~Hz}]$ vary in phase and amplitude. The $\mathrm{CD}$ drives have certain worst-case behaviors according to the specifications in the data sheet.

\section{Nominal and uncertainty models}

The resulting bode plots can be mapped onto the Nyquist plane. A straight forward method for obtaining the nominal and uncertainty models is to encircle the Nyquist plot of each frequency measurement which consists of 12 points, one for each $C D$ drive. This first set of circles are chosen to be of minimal area. A transfer function, called nominal model, $\mathrm{G}(\mathrm{s})$, is fitted to the center of the circles and the additive uncertainty model, $W_{A}(s)$, is then given by the second set of circles which contains the 12 points at the frequency in question and where the center is given by the fitted nominal model. The multiplicative uncertainty model, $W_{I}(s)$ is obtained by dividing the additive uncertainty model by the nominal model at every frequency point.

$$
G_{p}(s)=G(s)\left(1+W_{l}(s) \delta\right) \quad \delta \in \mathbf{C}, \quad|\delta| \leq 1 \quad \forall \omega
$$

$G_{p}$ represents the perturbed plant with multiplicative uncertainty given by the weighting function $W_{I}(s)$.
The second set of circles is obviously equal or of larger area than the first set. There are two main sources of conservatism with this method. The nominal model is a result of fitting the transfer function to the center of the circles. The lower the order, the higher the conservatism. The other source of conservatism is that circles yield a full-block complex perturbation uncertainty model, see eq. 1 . The Nyquist plot of each frequency measurement which consists of 12 points rarely has a circular shape distribution and an encirclement of the points will introduce certain conservatism as the uncertainty model will contain plants which are not physically possible. Fig. 2 shows the Nyquist plot of the 12 $\mathrm{CD}$ drives with the obtained nominal and uncertainty model.

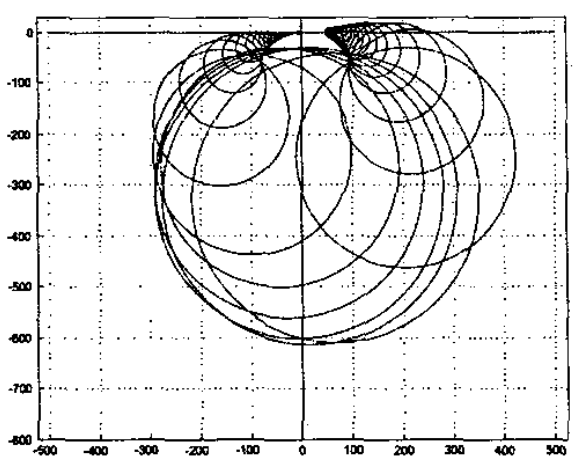

Figure 2: Second set of circles.

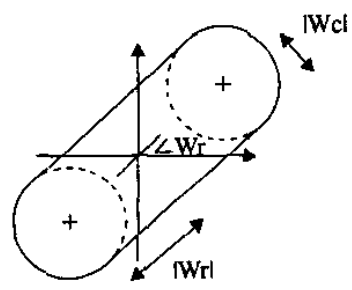

Figure 3: Frequency domain representation of the perturbation set.

In order to reduce the conservatism another geometric figure is considered in this paper, which is composed by a rectangle and two semicircles, one at each end of the smallest side of the rectangle. see fig. 3 . This geometric figure can mathematically be described by a real and a complex perturbation, see eq. 2.

$$
\begin{array}{r}
G_{p}(s)=G(s)\left(1+W_{c}(s) \delta_{c}+W_{r}(s) \delta_{r}\right) \\
\delta_{c} \in \mathbf{C}, \delta_{r} \in \mathbf{R}, \quad\left|\delta_{c}\right| \leq 1,-1 \leq \delta_{r} \leq 1
\end{array}
$$

where $W_{c}(s)$ and $W_{r}(s)$ are the complex and real perturbation weights respectively. For convenience the geometric figure is denoted olympic stadium (OS). Two main relevant methods can be distinguised for choosing OSs. The first one is choosing a minimal area OS, which at first sight may be the least conservative solution. The second one, treated in the sequel, is choosing the narrowest OS. Certain geometric 
calculations show that the narrowest $O S$ reduces the conservatism compared to a circle if the large side of the rectangle is $\frac{4}{\pi}$ times larger than the smallest side.

\section{Finding the first set of OSs}

In order to calculate the narrowest OS, first the convex hull of the set of Nyquist points for each frequency is determined. This convex hull will be a convex polyhedron with a subset of all the points observed as its vertices. The process of finding these vertices is shown in the upper subfigures of fig. 4. Once the polyhedron is determined, the distance between every side and its "most distant" point is calculated. A parallel line to the side in question containing its "most distant" point is traced and the remaining vertices must lay in between in order to ensure that the OS will contain all the points. If not all the vertices lay in between, the "most distant" point in question is not valid. The side with its closest valid "most distant" point is chosen and a parallel line to the chosen side is traced, which contains its closest valid "most distant" point. The sides of the OS are then found, see lower left fig. 4. The half of the distance between the two parallel lines determines the radius of the semicircles, $W_{c}$. These semicircles are moved in the same direction as the parallel lines towards the polyhedron until a vertix of the polyhedron is contained by the semicircles, see lower right fig. 4 . The half of the distance between the radii of the semicircles is $W_{r}$, and $\angle W_{r}$ is the angle between the principal axis of the $\mathrm{OS}$ and the shown coordinate system.
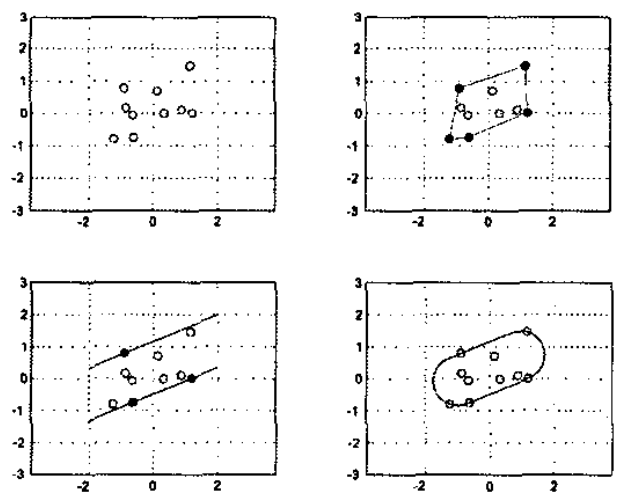

Figure 4: Calculating the first set of narrowest OSs.

Once the first set of OSs is found, a rational transfer function is fitted to the center of the OSs yielding the nominal model. As a result of the fit, the center of the OSs will no necessarily coincide with the nominal model and therefore a second set of OS must be found in order to center them according to the nominal model.

\section{Finding the second set of OSs}

The algorithm to find the second set of OS is slightly more involved since the center for each OS (which is not necessarily inside the polyhedron) is given by the nominal model. First the convex polyhedron must be determined, which is in fact the same polyhedron as for the first set of OSs, (a translation of the Nyquist points, as a result of the fit, does not alter the shape of the polyhedron). There are now two candidates which yield the narrowest OS: One side of the OS contains at least two vertices of the polyhedron. The second canditate is when the sides of the OSs contain at least one vertix each. For the shake of clarity, this is illustrated in fig. 5. The first case is shown in the two upper subfigures. Consider a polyhedron given by 3 vertices where the given center (without loose of generality) lays outside, see left upper subfigure. For each side, the distance with respect to the given center is calculated and the side where the given center is the closest valid "most distant" point is selected. A parallel line to the selected side is traced such that the given center is exactly in the middle, see right upper subfigure. In the second case, shown in the two lower subfigures of fig.5, where the center is also outside the polyhedron, it is clear that if the same method is applied as in the first case, it will not yield the narrowest OS. Instead all the median points must be calculated, represented as dots $\left(m_{1}, m_{2}\right.$ and $\left.m_{3}\right)$ in the right lower subfigure. A line from the given center to a median point is traced. If the "most distant" points of the median point are its corresponding two vertices, the median point is valid (in order to ensure that the OS will contain all the points). This procedure is repeated for all median points. The median point which has the closest "most distant" vertices is then selected yielding the narrowest OS. In the right lower subfigure, according to the described procedure, $m_{3}$ is selected and as a result the narrowest OS is found.

As it can be seen, finding the narrowest OS where the center is given, implies the investigation of both cases and the narrowest $O S$ among both cases is selected. Once the sides of the OS are found, the two semicircles can be found as described in the method for finding the first set of OSs.
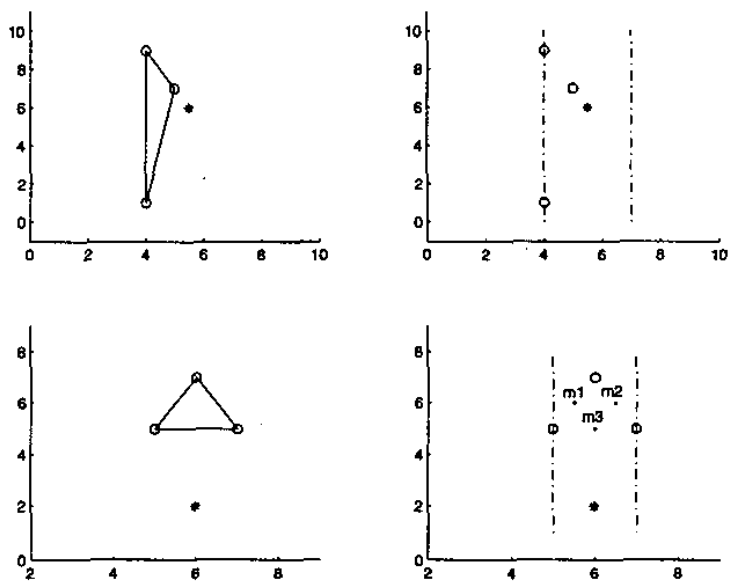

Figure 5: Calculating the 2nd set of narrowest OSs.

The resulting OS for the CD-drives are shown in fig. 6 . Even though the OSs cover a larger area in the Nyquist plane compared to the circles, the formers are narrower, an 


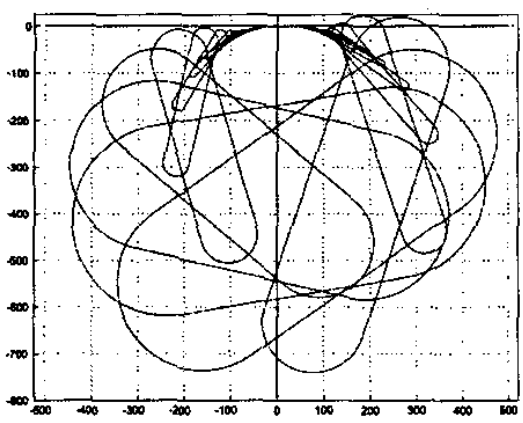

Figure 6: Second set of OS.

important property which can be exploited by algorithms for mixed- $\mu$ synthesis.

In order to find the multiplicative complex uncertainty weight, $W_{c}(s)$, a rational transfer function is fitted to the radii of semicircles constituting the OSs divided by the nominal model $G(s)$. Only magnitude is fitted as the phase is not relevant for complex uncertainties. Finding the multiplicative real uncertainty weight implies fitting a rational, stable, minimum phase in both magnitude and phase, which is not always trivial. When it comes to the controller synthesis, the uncertainty weights should generally be fitted to a low order transfer function.

\section{Deterministic method}

The deterministic method for obtaining the nominal and uncertainty models can therefore be summarized as follows:

- Find the 1st set of narrowest OSs at each frequency point which contains all the obtained Nyquist points.

- Fit a rational transfer function to the center of the 1 st set of $O S$ yielding the nominal model G(s).

- Find the 2nd set of narrowest OSs with center described by $G(s)$ containing at the same time all the obtained points at the corresponding frequency point.

- Fit a rational transfer function to the OS's width from the 2nd set divided by the nominal model, yielding the multiplicative complex uncertainty description $W_{c}(s)$, (only the magtinude is fitted).

- Fit a rational stable and minimum phase transfer function to OS's length from the 2nd set divided by the nominal model, yielding the multiplicative real uncertainty description $W_{r}(s)$.

Fig. 7 shows the center of the first set of OSs (solid line) and the fitted nominal model, $G(s)$, (dashed). Due to the accurate fit it can be hard to discern the bodeplots.
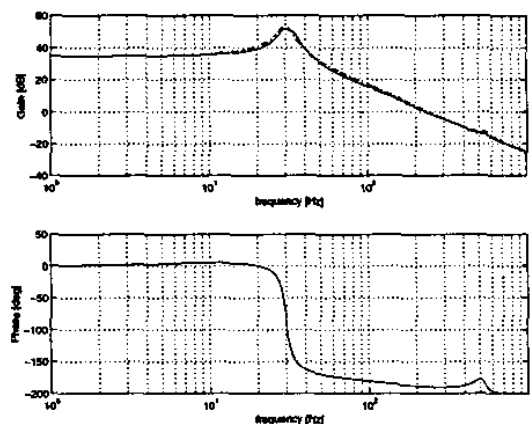

Figure 7: OS's center of 1st set (solid) and fitted nominal model (dashed).

Fig. 8 shows the measured and fitted multiplicative complex perturbations. It can be observed a step in the magnitude at $100[\mathrm{~Hz}]$ due to the a difference in the accuracy of the the methods used to measure the uncertainties. It is not chosen to fit the higher order dynamics around 500 in order to preserve a low order uncertainty weight at the expenses of a slighty more optimistic uncertainty weight.

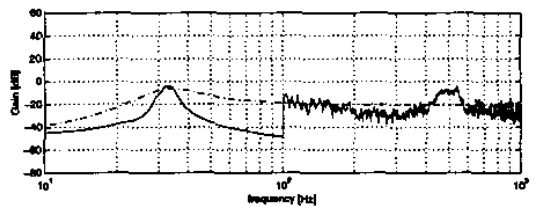

Figure 8: Measured and fitted mutiplicative complex perturbations.

Fig. 9 shows the measured and fitted multiplicative real perturbations. It is not chosen to fit the phase variations around $30[\mathrm{~Hz}]$ as it is not realistic that the mixed- $\mu$ algorithm will synthesize a controller where the Nyquist plot of the open loop will precisely surround -1 at $30[\mathrm{~Hz}]$
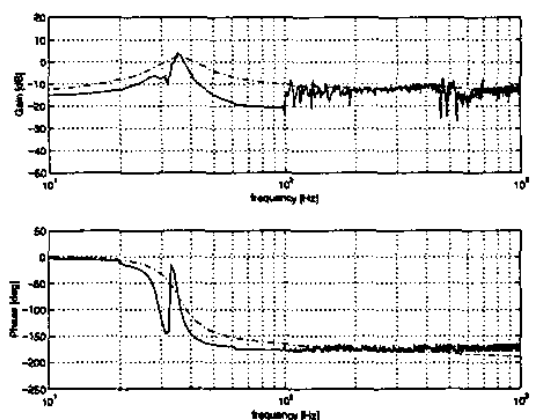

Figure 9: Measured and fitted mutiplicative real perturbations. 


\section{$4 \mu$-synthesis}

All the existing algorithms for $\mu$-synthesis are based on $\mathcal{H}_{\infty}$ synthesis, see e.g. [DGKF89], although considerably more complex and numerically involved. As mentioned above, a synthesis procedure based on the uncertainty model proposed in this paper relies on mixed $\mu$-synthesis, i.e. optimization for systems with both complex and real uncertainties. Examples of algorithms for mixed $\mu$-synthesis are [YD90, YND92] and [TCASN95]. The latter one, which involves solving a series of scaled DK-iterations is used in the sequel.

Fig. 10 depicts the control configuration with structured multiplicative input uncertainty. The task of the positioning controllers in $\mathrm{CD}$ players is to minimize the distance between the position of the focus point, which is the output of the plant, $G(s)$, and the position of the signal layer, $w_{1}$, where the information is contained. The performance weight, $W_{p 1}(s)$, which is the inverse of the desired sensitivity function is therefore placed at the output. $W_{p 2}$ can be interpreted as a complementary sensitivity weight, which in this configuration has been placed before the plant to control. $\varepsilon_{2}$ is a suitable small quantity such that the interaction between the disturbances $w_{1}$ and $w_{2}$ is reduced.

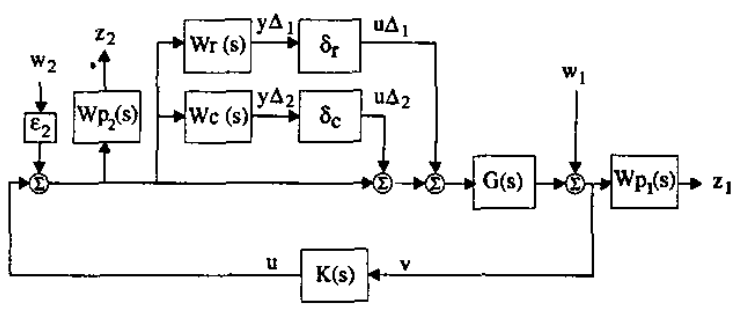

Figure 10: Control configuration with structured multiplicative input uncertainty.

The entire open loop system can be represented as a partioned matrix, as shown in eq. 3 ,

$$
P(s)=\left[\begin{array}{lll}
P_{11} & P_{12} & P_{13} \\
P_{21} & P_{22} & P_{23} \\
P_{31} & P_{32} & P_{33}
\end{array}\right]
$$

where the input is a column vector $\left[u_{\Delta} w u\right]^{T}$ and the output is also a column vector given by $\left[y_{\Delta} z v\right]^{T}$. The perturbations are "pulled-out" of the system and are included in a blockdiagonal matrix, $\Delta$, normalized such that $\|\Delta\|_{\infty} \leq 1$. The task of the algorithm for mixed $-\mu$ synthesis is to find a stabilizing controller $K$, which satisfies following conditions, where $N(s)$ is the result of the lower fractional transformation of the plant and the controller, $N(s)=F_{l}(P, K)$. (NS stands for nominal internal stability).
1. Nominal performance:

$$
N P: \quad \bar{\sigma}\left(N_{22}\right)=\mu_{\Delta_{P}}<1, \quad \forall \omega, \quad \text { and } \quad N S
$$

2. Robust stability:

$$
R S: \mu_{\Delta}\left(N_{11}\right)<1, \quad \forall \omega, \quad \text { and } N S
$$

3. Robust performance:

$$
R P: \mu_{\bar{\Delta}}(N)<1, \quad \forall \omega, \tilde{\Delta}=\left[\begin{array}{cc}
\Delta & 0 \\
0 & \Delta_{P}
\end{array}\right] \text { and } N S
$$

$\Delta_{P}$ is a full complex matrix used for analysis of robust performance.

\section{Simulations}

The control configuration in fig. 10 was utilized to synthesize two controllers, one with the mixed- $\mu$ algorithm, and the second with the DK-iteration algorithm which handles complex perturbations. A maximum order of 7 th degree was chosen for the D-scales fitting and the best controller, in terms of robust performance, was selected among 10 iterations. The results are listed below:

\begin{tabular}{|c|c|c|}
\hline & mixed- $\mu$ & DK \\
\hline NP & 0.49 & 0.81 \\
\hline RS & 0.81 & 0.75 \\
\hline RP & 0.96 & 1.19 \\
\hline
\end{tabular}

Table 1: Comparison between the mixed $\mu$ and DK controller

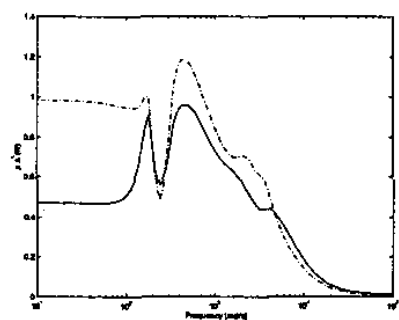

Figure 11: Robust performance for mixed $-\mu$ (solid) controller and $D K$ controller(dashed)

As it can be seen from the list, it was not possible to find a controller with the DK-iteration algorithm which meets the robust performance requirement. Fig. 11 shows a plot of $\mu \hat{\Delta}(N)$ along frequency. The DK-iteration algorithm does only handle complex perturbations and treats the "olympic stadiums" as circles leading inevitably to a more conservative design of the controller. However the mixed- $\mu$ algorithm is able to take benefit of the shape of the "olympic stadiums" and turns them such that the perturbed plant is furthest away from the instability point. 
A simulation of both controllers was performed in order to illuminate the difference in performance between both controllers. It was chosen to simulate the focus loop with the nominal plant. The disturbance $w_{2}$ was removed and $w_{1}$, which is the position of the signal layer of the $C D$ was composed by a sinusoid where the rotation of the $C D$ was set to $5.55[\mathrm{~Hz}]$. It was assumed that the vertical deviation of the CD was $50 \mu \mathrm{m}$ with a second harmonic of less amplitude, $7 \mu \mathrm{m}$. Furthermore, some frequency limited noise was added such that the simulation was more realistic. The upper subfigures in fig. 12 show the focus error, to the left, and the control signal, to the right of the mixed- $\mu$ controller. The focus error of the DK controller is shown in the left lower subfigure and its control signal in the right lower subfigure. It can be clearly appreciated that the mixed- $\mu$ controller can better cope with the disturbances as a result of a less conservative synthesis of the controller.
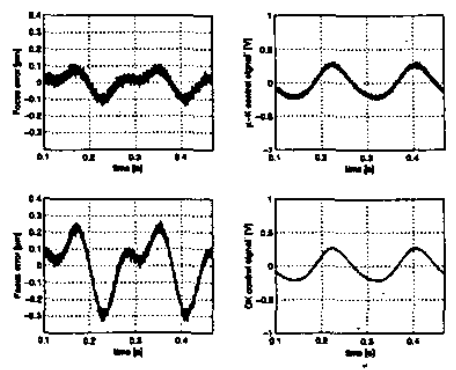

Figure 12: $\mu-K$ (upper subfigures), $D K$ (lower subfigures).

\section{Conclusion}

In this paper, a deterministic uncertainty modeling procedure has been proposed. The procedure has been applied to data for a number of $\mathrm{CD}$ drives with varying dynamical properties.

One of the characteristics of the system in this case study is that it has a resonancy which can vary from specimen to specimen. Using unstructured uncertainty descriptions to capture this type of variation, invariably leads to conservative results. The main reason for this is that there always will be a phase uncertainty at the average resonance frequency of close to 180 degrees. This means that the smallest circle (unstructured uncertainty description) that will cover all occurences will have a diameter of close to twice the amplitude of the resonance peak. This is in huge contrast to the actual robustness problems involved - in fact a very large gain can (and should) be used in a wide range of frequencies throughout the resonant area.

Although the proposed method still uses a 'black box' approach to uncertainty modeling, the obtained results shows that this problem with resonance peaks can be largely alleviated by the modeling procedure suggested.

In fact, it is the belief of the authors, that the conservatism involved with uncertainty modeling of resonant systems can almost be removed, if an iterative loop is applied to the synthesis. The idea would be to rotate the 'olympic stadiums' in such a way that the system and the compensator together would place observed points of uncertainty close to the stability margin. In that case, no performance would need to be sacrified at the cost of overly robustness.

Needless to say, deterministic uncertainty modeling requires that measurements can be obtained that grid the uncertainty space appropriately. If the measurements are too sparse, such a method might need to be complemented by some statistical extrapolation. In the case study presented, though, it turned out that considering a few well-chosen samples of different drives could provide a fairly precise uncertainty model.

\section{References}

[Det01] M. Dettori. LMI techniques for control with applications to a compact disc player mechanism. $\mathrm{PhD}$ thesis, Delft University, The Netherlands, 2001.

[DGKF89] J. Doyle, K. Glover, P. Khargonekar, and B.A. Francis. State-space solutions to standard $\mathcal{H}_{2}$ and $\mathcal{H}_{\infty}$ control problems. IEEE Transactions on Automatic Control, AC-34:831-847, 1989.

[Lee98] Chak Hong Lee. Robust repetitive control and application to a $C D$ player. CRC Press, Ed: W.S. Levine, Trinity Hall Cambridge, february 1998. A dissertation submitted for the degree of Doctor Philosophy.

[PGRV92] R. Pintelon, P. Guillaume, Y. Rolain, and F. Verbeyst. Identification of linear systems captured in a feedback loop. IEEE Transactions on Instrumentation and Measurement, 41(6):14-20, december 1992.

[SSB96] M. Steinbuch, G. Schootstra, and O.H. Bosgra. Robust Control of a Compact Disc Mechanism, chapter Applications of control. CRC Press, Ed: W.S. Levine, 1996.

[TCASN95] S. Tøffner-Clausen, P. Andersen, J. Stoustrup, and H.H. Niemann. A new approach to $\mu$-synthesis for mixed perturbation sets. In Proceedings of the European Control Conference, pages 147-152, sep 1995.

[VSA $^{+} 01$ ] E. Vidal, J. Stoustrup, P. Andersen, T.S. Pedersen, and H.F. Mikkelsen. Open and closed loop parametric system identification in compact disk players. In Proceedings of the 1999 American Control Conference, 2001.

[YD90] Peter M. Young and John C. Doyle. Computation of $\mu$ with real and complex uncertainties. In Proceedings of the 29th Conference on Decision and Control, pages 12301235, Honolulu, Hawaii, december 1990.

[YND92] Peter M. Young, Matthew P. Newlin, and John C. Doyle. Practical computation of the mixed $\mu$ problem. In Proceedings of the American Control Conference, pages 2190-2194, 1992.

[YP00] Ting-Jen Yeh and Yi-Chuan Pan. Modeling and identification of opto-mechanical coupling and backlash nonlinearity in optical disk drives. IEEE Transactions on Consumer Electronics, 46(1): 105-115, february 2000. 